This article was downloaded by: [180.211.214.189]

On: 24 May 2014, At: 05:53

Publisher: Taylor \& Francis

Informa Ltd Registered in England and Wales Registered Number: 1072954 Registered office: Mortimer House, 37-41 Mortimer Street, London W1T 3J H, UK

\title{
Brain-Computer Interfaces
}

Publication details, including instructions for authors and subscription information: http:// www. tandfonline.com/loi/tbci20

\section{Affective brain-computer interfaces: Special Issue editorial}

\author{
Christian Mühl Ph.D. ${ }^{a}$, Brendan Allison Ph. D. ${ }^{b}$, Anton Nijholt Ph. D. ${ }^{\text {cd }} \&$ Guillaume Chanel \\ Ph. D. ${ }^{\text {e }}$ \\ ${ }^{a}$ Inria Bordeaux - Sud-Ouest, Talence, France \\ ${ }^{\mathrm{b}}$ ASPEN Lab, Electrical and Computer Engineering Department, Old Dominion University, \\ Norfolk, VA, USA \\ ${ }^{c}$ Department of Cognitive Science, University of California at San Diego, La J olla, CA, USA \\ ${ }^{d}$ Faculty EEMCS, Human Media Interaction, University of Twente, Enschede, The \\ Netherlands \\ e Swiss Center for Affective Sciences, Campus Biotech, CISA - University of Geneva, Genève, \\ Switzerland \\ Published online: 22 May 2014.
}

To cite this article: Christian Mühl Ph.D., Brendan Allison Ph.D., Anton Nijholt Ph.D. \& Guillaume Chanel Ph.D. (2014) Affective brain-computer interfaces: Special Issue editorial, Brain-Computer Interfaces, 1:2, 63-65, DOI: $10.1080 / 2326263 \times .2014 .913829$

To link to this article: http:// dx. doi.org/ 10.1080/2326263X.2014.913829

\section{PLEASE SCROLL DOWN FOR ARTICLE}

Taylor \& Francis makes every effort to ensure the accuracy of all the information (the "Content") contained in the publications on our platform. However, Taylor \& Francis, our agents, and our licensors make no representations or warranties whatsoever as to the accuracy, completeness, or suitability for any purpose of the Content. Any opinions and views expressed in this publication are the opinions and views of the authors, and are not the views of or endorsed by Taylor \& Francis. The accuracy of the Content should not be relied upon and should be independently verified with primary sources of information. Taylor and Francis shall not be liable for any losses, actions, claims, proceedings, demands, costs, expenses, damages, and other liabilities whatsoever or howsoever caused arising directly or indirectly in connection with, in relation to or arising out of the use of the Content.

This article may be used for research, teaching, and private study purposes. Any substantial or systematic reproduction, redistribution, reselling, loan, sub-licensing, systematic supply, or distribution in any form to anyone is expressly forbidden. Terms $\&$ Conditions of access and use can be found at http:// www.tandfonline.com/page/terms-and-conditions 


\section{Editorial}

\section{Affective brain-computer interfaces: Special Issue editorial}

\section{Introduction}

Over the last several years, brain-computer interface (BCI) research has grown well beyond initial efforts to provide basic communication for people with severe disabilities that prevent them from communicating otherwise. Since BCIs rely on direct measures of brain activity, users do not have to move in any way to convey information.[1] During the early years of BCI research, BCI systems had little to offer healthy users. Since most users can communicate quickly and easily by speaking or typing, why would healthy people use a $\mathrm{BCI}$ ?

Several different answers to this question have been presented. For example, when healthy users' hands and/ or voice are not available in certain situations, they may experience 'situational disability'. In that situation, healthy users may need a low-bandwidth interface. Healthy people may use a BCI just for fun; the idea of communicating just through brainwaves is new and exciting to many people. BCIs may also detect emotion, arousal, or other characteristics that would otherwise be burdensome, irritating, or impossible to convey through other means.[2-4]

The last category of BCIs presented includes 'affective BCIs' or 'aBCIs', which are BCIs that detect affect. Information about users' affect could be used to modify the user's interaction with software and people in real time. For example, the alpha WoW system can change a user's World of Warcraft avatar if the user's EEG indicates an increased level of stress.[5] Users could always press a button to send this information, but doing so creates some distraction and burden just when the user exhibits stress, and may need hands free to deal with ingame challenges. Similarly, games or other software applications might automatically adapt to states when users seem overburdened or bored.[6,7] Again, users could choose to press a button when they want to increase or decrease their workload, but might prefer to keep focused on their work while the environment adapts automatically.

A related approach uses BCI technology to detect confusion, frustration, or the user's belief that an error has just occurred. Users might inform their software application through manual means, such as a backspace key or clicking an icon to repeat any recent feedback. This manual communication could burden the user when s/he is already annoyed or confused, whereas an aBCI could automatically initiate changes that help the user focus on accomplishing goals.

Tools to monitor drowsiness based on the EEG or other means have been explored for many decades, but recent work has made such systems far more practical. For example, a driver might wear a headband that detects alertness from the EEG. If the driver begins to fall asleep, a cell phone might sound an alarm or initiate other actions. This approach might be useful for other people in attention-critical situations such as pilots, security guards or nuclear plant operators. In this case, the aBCI provides information that might be unavailable otherwise; presumably, a person who is falling asleep was not planning on pressing a button to convey this dangerous change.

Affective data could also be analyzed offline to provide additional information to researchers, market researchers, software designers, and quality assurance personnel. The relatively new field of neuromarketing highlights different advantages that affective monitoring can provide. Companies may want the most detailed information possible about people's affective responses to advertisements, movie trailers, political speeches, etc. People who watch material and then comment later may forget their reactions to specific scenes, change their minds, or provide only limited details of their reactions. Subjects participating in focus groups might change or omit their true reactions to please a focus-group moderator or avoid conflict with vocal focus-group subjects. Affective monitoring tools could provide rich, multidimensional information about each viewer's reactions immediately, without distracting subjects or pausing the material they are expected to view. This information might be available in real time, essentially providing a decision-support system that could help focus-group moderators decide which material to present next or ask subjects for more information.

Many other applications of aBCI technology have been proposed, and often implemented and sold. An alarm clock might sense when users are in certain sleep stages and trigger an alarm a little early instead of awaken them from deep sleep. Related technology could help identify the best times to study, relax, write, nap, etc. Software might help treat emotional disorders or encourage wellness, relaxation or creativity. Tools could 
detect when users are 'in the zone', either by themselves or as part of collaborative efforts, perhaps encouraging synergy among users.[8] Our survey paper in this Special Issue presents further studies and discussion. These are only a few examples to highlight some promising directions and illustrate the breadth of opportunity available. These examples also help explain our interest in encouraging new research through our Special Issue.

\section{Special Issue contributions}

On 2 September 2013, we hosted the 3rd Workshop on Affective Brain-Computer Interfaces $(\mathrm{aBCI})$, along with the ACII 2013 conference in Geneva, Switzerland from 2 to 5 September. We were very impressed with many of the presentations, and some attendees were interested in publishing extended and updated versions of their work. We felt that a Special Issue in the new BCI Journal would be an excellent venue.

This Special Issue presents six articles devoted to aBCIs. Over a dozen articles were submitted to this Special Issue, and we editors faced a serious challenge deciding on the best articles. All articles were peerreviewed by three experts and scored on several axes such as originality and scientific contribution. Then, the top articles were returned to their authors to implement changes suggested by the reviewers, and the resulting articles were reviewed again before final acceptance. Hence, the articles presented here reflect a rigorous peerreview procedure and reflect the most promising ideas, methods, and results from the aBCI community.

The Special Issue begins with 'A survey of affective brain computer interfaces: principles, state-of-the-art, and challenges'. We, as guest editors provide a broad overview of neurophysiology-based affect detection, from its underlying psychological concepts to its neuroscientific foundations. We discuss the state-of-the-art and possible applications within the BCI ecosystem, technical and methodological standards, and challenges that must be overcome to advance toward reliable, practical aBCI systems.

The article by Kroupi and colleagues titled 'Implicit affective profiling of subjects based on physiological data coupling' explores the relationship existing between EEG signals and electrodermal activity (EDA) and its potential application toward aBCI. This relationship is analyzed by measuring the amount of phase synchrony between the EEG envelope and EDA while the participants were watching emotional music clips. The results demonstrate that this measure of synchrony, particularly in the temporal lobe, could be employed as a new feature for emotion recognition. Furthermore, the authors propose a method to profile the subjects based on EEG/ EDA synchrony, which is able to cluster subjects according to their emotional ratings.
In 'EEG-based classification of positive and negative affective states', Stikic and colleagues present an aBCI study on the continuous assessment of emotions elicited by videos and its application to the prediction of charity donation. This study analyzes the aBCIs ability to generalize by employing a high number of participants (more than 150) and by testing the models on sessions, participants and videos different than those used for training. The results demonstrate that the aBCIs were able to generalize well with respect to the participants and the sessions, but did not generalize as well across different types of videos. The authors also show the potential of $\mathrm{aBCI}$ and continuous emotion assessment to predict charity donations after viewing a moral (or amoral) story telling session.

Heger and colleagues, in their paper titled 'Continuous affective states recognition using functional near infrared spectroscopy', present an affect recognition system that uses metabolic signals measured from prefrontal cortices to classify affective states induced by visual and auditory stimulation. Unlike more conventional, eventrelated approaches, they classify based on time-domain and wavelet-based signal features extracted from the functional near infrared spectroscopy (fNIRS) signals in a quasi-continuous manner, thereby allowing a dynamic affect detection with a high temporal resolution.

The article 'Affective brain-computer interfaces as enabling technology for responsive psychiatric stimulation' by Widge and colleagues first defines the limitations of passive aBCI for the control of affective prostheses designed to stimulate patients suffering from affective disorders. The authors then propose to employ prefrontal cortex activity to infer the patients' voluntary intent to regulate their emotions. This proposition is tested on rats, which were implanted with a medial forebrain (MFB) stimulator controlled by prefrontal cortex signals. The results demonstrate the feasibility of this approach and could inspire new therapies using active aBCIs.

The article 'Using functional near infrared spectroscopy to measure moral decision-making: effects of agency, emotional value, and monetary incentive' by Strait and Scheutz presents an experimental study to investigate the effects of several factors, namely agency, emotional value, and monetary incentive, in moral decision-making. The authors use fNIRS to measure hemodynamic activity. In particular, they explore the prefrontal cortex (PFC), which has been identified through fMRI and other studies as central to moral decision-making. fNIRS may provide an alternative to fMRI for observing the decision-making process. The findings show which of the varying factors significantly affect the measurements of the left and which of the right PFC. Interestingly, they also show that monetary incentives do have an effect on the hemodynamic activity. This suggests that an increased monetary incentive might have influence on behavior that fNIRS can assess. 


\section{Discussion and conclusion}

These articles, and other examples, share an underappreciated characteristic of aBCIs: they could help people. BCI research has long been rooted in altruism. Most early efforts sought to provide communication for people who might otherwise be unable to move, and newer directions aim to help people recover from stroke, autism, and other conditions. The work presented here may lead to altruistic applications, such as helping persons with psychiatric disorders, promoting relaxation and personal wellness, encouraging charitable donations, or elucidating moral decision-making processes.

Some aBCI applications are many years away from helping people. For example, as Widge and colleagues note in their article, their initial tests with rats present an initial proof of concept, and would require extensive additional development before even beginning initial clinical trials with people. However, many other directions may be relatively close to market, catalyzed by more portable, practical, comfortable, and powerful tools to record the EEG.[9,10] Companies have been selling systems called 'BCIs' for home use for a several years, which often incorporate affective information into games and help buyers monitor concentration or relaxation. Noninvasive systems that do not require medical certification and rely on inexpensive, practical electrodes could get aBCI technologies to users fairly quickly.

This rapid progress also raises ethical concerns for some aBCI applications. Systems designed to record, classify and even change affective state may entail safety, privacy, and security issues.[3,8,11] As with passwords and other private information, people should follow commonsense guidelines, such as keeping data private as much as possible. If data are stored offline for later analysis, in raw or processed form, confidentiality is essential. Multiplayer aBCI games and other applications that share data online should be secure. Even if raw or processed EEG data are kept on users' local systems, derivative information like 'the character changed to a bear' could reveal when users are under stress. Testing with human subjects, when appropriate, should follow normative ethical guidelines.

The studies presented in this Special Issue help identify and elaborate new directions and opportunities in aBCI research. These and other studies show that aBCI technology is rapidly maturing, with novel ideas and improved technologies that could encourage new research directions and products. We thank the authors for their articles, and hope the next few years of aBCI research continues to produce conference workshops, articles and products with new, reasonable, and helpful tools.

\section{References}

[1] Wolpaw JR, Wolpaw EW. Brain-computer interfaces: principles and practice. Oxford: Oxford University; 2012.

[2] Allison BZ, Graimann B. Why use a BCI if you're healthy? Trends Controversies: Brain-Comput Interfac Intell Syst, IEEE Intell Syst. 2008;23:76-78.

[3] Allison BZ, Dunne S, Leeb R, Millan J, Nijholt A. Recent and upcoming bci progress: overview, analysis, and recommendations. In: Allison BZ, Dunne S, Leeb R, del Millan JR, Nijholt A, editors. Towards practical BCIs: bridging the gap from research to real-world applications. Berlin: Springer-Verlag; 2013. p. 1-13.

[4] van de Laar B, Gürkök H. Plass-Oude Bos B, Nijboer F, Nijholt A. User experience evaluation of brain-computer interfaces. In: Allison BZ, Dunne S, Leeb R, del Millan JR, Nijholt A, editorsTowards practical brain-computer interfaces: bridging the gap from research to real-world applications. Berlin: Springer-Verlag; 2013. p. 223-237.

[5] Plass-Oude Bos D, Reuderink B, van de Laar B, Gürkök H, Mühl C, Poel M, Nijholt A, Heylen D. Brain-computer interfacing and games. In: Tan D, Nijholt A, editors. Brain-computer interfaces: applying our minds to humancomputer interaction. London: Human-Computer Interaction Series, Springer-Verlag; 2010. p. 149-178.

[6] Fairclough SH. Fundamentals of physiological computing. Interact Comput. 2009;21:133-145.

[7] Chanel G, Rebetez C, Bétrancourt M, Pun T. IEEE transactions on systems man and cybernetics. Part A: Syst Humans. 2011;41:1052-1063.

[8] Gürkök H, Nijholt A, Poel M, Obbink M. Evaluating a multiplayer brain-computer interface game: challenge versus coexperience. Entertain Comput. 2013;4(3):195-203.

[9] Chi YM, Wang YT, Wang Y, Maier C, Jung TP, Cauwenberghs G. Dry and noncontact EEG sensors for mobile brain-computer interfaces. IEEE Trans Neural Syst Rehabil Eng. 2012;20:228-235.

[10] Guger C, Krausz G, Allison BZ, Edlinger G. A comparison of dry and gel-based electrodes for P300 BCIs. Frontiers Neurosci. 2012;6:60.

[11] Fairclough SH. Physiological data should remain confidential. Nature. 2013;505:263.

Christian Mühl, Ph.D.

Inria Bordeaux - Sud-Ouest, Talence Cedex, France

Brendan Allison, Ph.D.

ASPEN Lab, Electrical and Computer Engineering

Dept., Old Dominion University, Norfolk, VA, USA and

Department of Cognitive Science, University of California at San Diego, La Jolla, CA, USA

Anton Nijholt, Ph.D.

University of Twente, Faculty EEMCS, Human Media Interaction, Enschede, the Netherlands

Guillaume Chanel, Ph.D.

Swiss Center for Affective Sciences, CISA - University of Geneva, Geneva, Switzerland 